\title{
A SEPARATION THEOREM FOR MANIFOLDS
}

\author{
RUSSELL G. BRASHER
}

Let $X$ be a compact connected $n$-manifold without boundary. We assume a cohomology theory satisfying the continuity axiom as well as the Eilenberg-Steenrod axioms and defined on a class of spaces which includes the compact manifolds, e.g., the Alexander theory on the category of compact pairs [3, Chapter 6 , $\$ 4$ et seq.]. The coefficient group throughout will be the additive group $Z$ of integers if $X$ is orientable and the group $Z_{2}$ of integers mod 2 if $X$ is nonorientable. For standard results in the algebraic treatment of manifolds one may consult Eilenberg-Steenrod [1, Chapter 11, §6] or Spanier [3, Chapter 6]. We denote the $q$-dimensional cohomology group of $X$ by $H^{q}(X)$; if $q=0, H^{q}(X)$ is taken to be the reduced zero-dimensional group.

For a closed proper subset $A$ of $X$ let $i: A \rightarrow X$ be the inclusion map. The purpose of this note is to prove the following theorem.

THEOREM 1. The subset $A$ separates $X$ if and only if

$$
i^{*}: H^{n-1}(X) \rightarrow H^{n-1}(A)
$$

is not surjective.

We shall need some preliminary notions and results; for details see Wallace [4] or Keesee [2].

The inclusion $i: A \rightarrow X$ induces homomorphisms $i^{*}: H^{q}(X) \rightarrow H^{q}(A)$; if $e \in H^{q}(X)$ we shall denote $i^{*}(e)$ by $e \mid A$, and if $u \in H^{q}(A)$ is in the image of $i^{*}$ we shall say that $u$ can be extended to $X$.

Let $e \in H^{q}(X)$ and let $M$ be a closed proper subset of $X$ such that $e \mid M \neq 0$ but $e \mid N=0$ for every closed proper subset $N$ of $M$. Then $M$ is called a minimal support for $e$.

Let $A$ be a closed subset of $X$ and $e \in H^{q}(A)$. An irreducible membrane for $e$ is a closed subset $R$ of $X$ such that $e$ cannot be extended to $R \cup A$ but can be extended to $S \cup A$ for each closed proper subset $S$ of $R$.

We have the following results.

(i) There exists a minimal support for each nonzero element of $H^{q}(X)$. Each minimal support is connected.

(ii) If $e \in H^{q}(A)$ cannot be extended to $X$, then $e$ has an irreducible membrane $R$ in $X$. Furthermore, $e \mid(R \cap A)=e_{0} \neq 0, R$ is an irreducible

Received by the editors March 17, 1969. 
membrane for $e_{0}, R=(R-A)^{-}$, and $R-A$ is connected.

(iii) If $R$ is an irreducible membrane for an element in $H^{n-1}(A)$, then $R-A$ is open in $X$.

The proof of (iii) is similar to that in [2, Theorem 1.5].

The following lemma extends a familiar theorem for spheres to arbitrary manifolds. Recall that in a cohomology theory satisfying the continuity axiom on the category of compact pairs, every compact triad is proper and the associated Mayer-Vietoris sequence is exact.

Lemma 1. If $A$ is a closed proper subset of the n-manifold $X$, then $H^{n}(A)=0$.

Proof. Suppose $e \in H^{n}(A), e \neq 0$. Without loss of generality, we may assume that $A$ is a minimal support for $e$.

Since $A$ is not all of $X$, there is a point $x$ in the boundary of $A$ and we can find a coordinate nbd $U$ of $x$ whose boundary intersects $X-A$. The boundary of $U, \partial U$, is an $S^{n-1}$, and $\partial U \cap A=R$ is a closed proper subset of $S^{n-1}$; thus $H^{n-1}(R)=0$. Let $S=A-U, T=\bar{U} \cap A$; then $S \cap T=R$ and $S \cup T=A$. Consider the portion of the Mayer-Vietoris sequence of the triad $(A ; S, T)$ :

$$
\cdots \rightarrow H^{n-1}(R) \rightarrow H^{n}(A) \stackrel{j}{\rightarrow} H^{n}(S) \oplus H^{n}(T) \rightarrow \cdots .
$$

Since $H^{n-1}(R)=0, j$ is injective. Thus $j(e) \neq 0$. But $S$ and $T$ are closed proper subsets of $A$, and since $A$ is a minimal support for $e, e|S=e| T=0$, which implies that $j(e)=(e|S, e| T)=0$.

LeMma 2. If $R$ is an irreducible membrane for an element in $H^{n-1}(A)$, then $R-A$ is a component of $X-A$.

Proof. Since $R-A$ is connected, it is contained in some component $C$ of $X-A$. Suppose $R-A$ is not all of $C$. Then we have $C-R \neq \square$, $R-A \neq \square$, and $(R-A) \cup(C-R)=C$. Now $(R-A)^{-}=R$, so $(R-A)^{-}$ $\cap(C-R)=\square$. Since $R-A$ is open in $X$, it follows that $(C-R)^{-}$ $\cap(R-A)=\square$. But this implies that $C$ is not connected.

We are now ready to prove the separation theorem.

Theorem 2. $A$ separates $X$ if and only if $i^{*}: H^{n-1}(X) \rightarrow H^{n-1}(A)$ is not surjective.

Proof. (a) Suppose $A$ separates $X ; X-A=P \cup Q, P$ is separated from $Q, P$ and $Q$ nonempty. Let $R=A \cup P, S=A \cup Q$; then $R \cap S=A$ and $R \cup S=X$. Consider the portion of the Mayer-Vietoris sequence for $(X ; R, S)$ : 


$$
\begin{aligned}
& \cdots \rightarrow H^{n-1}(R) \oplus H^{n-1}(S) \stackrel{i^{\prime}}{\rightarrow} H^{n-1}(A) \stackrel{\delta}{\rightarrow} H^{n}(X) \stackrel{j^{\prime}}{\rightarrow} H^{n}(R) \\
& \oplus H^{n}(S) \rightarrow \cdots
\end{aligned}
$$

Now $i^{*}: H^{n-1}(X) \rightarrow H^{n-1}(A)$ surjective would imply $i^{\prime}$ surjective, $\delta=0$, and $j^{\prime}$ injective. This is impossible since $H^{n}(X) \neq 0$ and $H^{n}(R)$ $\oplus H^{n}(S)=0$.

(b) Now suppose $X-A$ is connected, and assume that $i^{*}$ is not surjective. Then there is an element $e \in H^{n-1}(A)$ that cannot be extended to $X ; e$ has an irreducible membrane $R$ in $X$.

By Lemma $2, R-A$ is a component of $X-A$ and thus $R-A$ $=X-A$. This implies that $R \cup A=X$.

Let $U$ be a coordinate nbd in $R-A$ such that $\bar{U}$ does not intersect $A$. Now, since $R$ is an irreducible membrane for $e, e$ can be extended to an element

$$
\bar{e} \in H^{n-1}(A \cup(R-U))=H^{n-1}(X-U) .
$$

The boundary of $U$ is an $S^{n-1}$; and $\bar{e} \mid S^{n-1} \neq 0$, for if it were, $e$ could be extended to $R \cup U$ (see [2, Theorem 1.2]). Consider the portion of the Mayer-Vietoris sequence for $(X ; \bar{U}, X-U)$ :

$$
\begin{aligned}
\cdots & \rightarrow H^{n-1}(\bar{U}) \oplus H^{n-1}(X-U) \stackrel{i^{\prime}}{\rightarrow} H^{n-1}\left(S^{n-1}\right) \stackrel{\delta}{\rightarrow} H^{n}(X) \\
& \rightarrow H^{n}(\bar{U}) \oplus H^{n}(X-U) \rightarrow \cdots
\end{aligned}
$$

Since the last group is trivial, $\delta$ is surjective. Thus

$$
H^{n-1}\left(S^{n-1}\right) / \operatorname{Im} i^{\prime} \approx H^{n}(X) .
$$

Now Im $i^{\prime} \neq 0$, since we know that $\bar{e} \mid S^{n-1} \neq 0$. If $X$ is orientable the isomorphism above becomes $Z_{n} \approx Z$ for some $n \neq 1$; if $X$ is nonorientable it becomes $H^{n}(X)=0$. In either case we have a contradiction.

Corollary 1. The subset $A$ separates $X$ if and only if

$$
j^{*}: H^{n}(X, A) \rightarrow H^{n}(X)
$$

is bijective.

We also obtain the following well-known characterization of sets that separate spheres [2].

Corollary 2. A closed proper subset $A$ of $S^{n}$ separates $S^{n}$ if and only if $H^{n-1}(A) \neq 0$.

The author wishes to thank the referee for his helpful suggestions. 


\section{BIBLIOGRAPHY}

1. S. Eilenberg and N. Steenrod, Foundations of algebraic topology, Princeton Univ. Press, Princeton, N. J. 1952.

2. J. W. Keesee, Sets which separate spheres, Proc. Amer. Math. Soc. 5 (1954) 193200.

3. E. Spanier, Algebraic topology, McGraw-Hill, New York, 1966.

4. A. D. Wallace, $A$ theorem on end points, An. Acad. Brasil. Ci. 22 (1950), 29-33.

Stephen F. Austin State College 